University of Nebraska - Lincoln

DigitalCommons@University of Nebraska - Lincoln

Faculty Publications, Department of Psychology

Psychology, Department of

$2-2004$

\title{
Serotonin Transporter and GABA(A) Alpha 6 Receptor Variants Are Associated with Neuroticism
}

\author{
Srijan Sen \\ University of Michigan - Ann Arbor \\ Sandra Villafuerte \\ University of Michigan - Ann Arbor \\ Randolph Nesse \\ University of Michigan - Ann Arbor \\ Scott F. Stoltenberg \\ University of Nebraska-Lincoln, sstoltenberg2@unl.edu \\ Jeffrey Hopcian \\ University of Michigan - Ann Arbor \\ See next page for additional authors
}

Follow this and additional works at: https://digitalcommons.unl.edu/psychfacpub

Part of the Psychiatry and Psychology Commons

Sen, Srijan; Villafuerte, Sandra; Nesse, Randolph; Stoltenberg, Scott F.; Hopcian, Jeffrey; Gleiberman, Lillian; Weder, Alan; Burmeister, Margit; and University of Michigan - Ann Arbor, "Serotonin Transporter and GABA(A) Alpha 6 Receptor Variants Are Associated with Neuroticism" (2004). Faculty Publications, Department of Psychology. 448.

https://digitalcommons.unl.edu/psychfacpub/448

This Article is brought to you for free and open access by the Psychology, Department of at DigitalCommons@University of Nebraska - Lincoln. It has been accepted for inclusion in Faculty Publications, Department of Psychology by an authorized administrator of DigitalCommons@University of Nebraska - Lincoln. 


\section{Authors}

Srijan Sen, Sandra Villafuerte, Randolph Nesse, Scott F. Stoltenberg, Jeffrey Hopcian, Lillian Gleiberman, Alan Weder, Margit Burmeister, and University of Michigan - Ann Arbor 


\title{
Serotonin Transporter and GABA(A) Alpha 6 Receptor Variants Are Associated with Neuroticism
}

\author{
Srijan Sen, ${ }^{\prime}$ Sandra Villafuerte, ${ }^{2}$ Randolph Nesse, ${ }^{3,4}$ Scott F. Stoltenberg, ${ }^{3,7}$ Jeffrey Hopcian, ${ }^{2}$ \\ Lillian Gleiberman, ${ }^{5}$ Alan Weder, ${ }^{5}$ and Margit Burmeister, ${ }^{1,2,3,6}$
}

\begin{abstract}
${ }^{1}$ Neuroscience Program; ${ }^{2}$ Mental Health Research Institute; ${ }^{3}$ Department of Psychiatry; ${ }^{4}$ Department of Psychology and Institute for Social Research; ${ }^{5}$ Department of Internal Medicine; ${ }^{6}$ Department of Human Genetics; ${ }^{7}$ Addiction Research Center, University of Michigan, Ann Arbor, Michigan, USA
\end{abstract}

Corresponding author - M. Burmeister, Mental Health Research Institute, 205 Zina Pitcher Place, University of Michigan, Ann Arbor MI 48I09-0720, USA

\begin{abstract}
Background: A tendency to experience negative affect, as measured by the neuroticism component of the Neuroticism, Extraversion, and Openness Personality Inventory (NEO-PI), is a trait marker for major depression. Epidemiologic studies indicate a strong genetic component, but to date few specific genetic variants have been definitively implicated. A serotonin transporter promoter polymorphism (5-HTTLPR) has been extensively studied in neuroticism and several psychiatric disorders, with inconclusive results. A GABA(A) receptor a6 subunit variant (Pro385Ser) has been associated with alcohol-related traits but has not been studied in neuroticism or depression.

Methods: A total of 384 subjects who completed the NEO-PI were genotyped at 5-HTTLPR and Pro385Ser. Associations between polymorphisms and both alcohol use and personality domains were tested.

Results: The 5-HTTLPR short allele $(p=.008)$ and Pro385Ser Pro allele $(p=.003)$ are associated with higher neuroticism scores. The 5-HTTLPR long allele $(p=.006)$, but not Pro385Ser, is also associated with an increased presence of alcohol use. In addition, there is a nonsignificant suggestion of an interaction: the effect of 5-HTTLPR on neuroticism might be dependent on the Pro385Ser genotype.

Conclusions: These findings support a role for the serotonin transporter and GABA(A) a6 subunit in depression-related traits.
\end{abstract}

Keywords: Serotonin, $\gamma$-aminobutyric acid, association, interaction, NEO-PI, personality, polymorphism, anxiety, depression

The principal function of the serotonin transporter is to remove serotonin from the synapse, returning it to the presynaptic neuron where the neurotransmitter can be degraded or retained for re-release at a later time. Heils et al. (1995) identified a functional repeat polymorphism in the promoter region of the serotonin transporter gene (5HTTLPR) where the 14-repeat (s) and 16-repeat (l) variants predominate in Caucasian populations. The same group found that cell lines with at least one 5-HTTLPR s allele produced about half as much of a reporter protein as cell lines homozygous for the 1 allele. The reduced efficiency of the s allele has been confirmed through messenger ribonucleic acid quantification in cell lines, postmortem human brain, and whole blood serotonin and platelet studies (Hanna et al., 1998; Lesch et al., 1996; Little et al., 1998). 5-HTTLPR subsequently became one of the most extensively investigated polymorphisms in studies of depression and its trait marker, neuroticism. Lesch et al. (1996) found that the presence of the 5-HTTLPR s allele was associated with higher mean neuroticism scores. This finding prompted many attempts to replicate the association. In four replication attempts, a sample size similar to that of the original study was used (>350 subjects) (Greenberg et al., 2000; Hamer et al., 1999; Jorm et al., 1998; Mazzanti et al., 1998). One study, carried out by the group that published the original association, robustly confirmed the initial association between 5-HTTLPR and neuroticism (Greenberg et al., 2000). A second study did not confirm the original association but found evidence for linkage between 5-HTTLPR and anxiety-related traits (Mazzanti et al., 1998). The other two studies found no evidence for either association or linkage (Hamer et al., 1999; Jorm et al., 1998). In addition to these larger studies, at least 19 studies with consid- erably smaller sample sizes have been conducted. Although some of these studies confirmed the original association, most did not (Greenberg et al., 2000; Melke et al., 2001). These results are not surprising, given the lack of statistical power of these studies. Our study investigates the 5-HTTLPR-neuroticism association with the use of a large sample and the same personality inventory that was used in the original study.

The $r$-aminobutyric acid (GABA) system has been repeatedly implicated in anxiety- and depression-related traits (Petty, 1995). Cerebrospinal fluid and occipital cortex GABA concentrations are reduced in depressed patients (Sanacora et al., 1999; Sanacora et al., 2000). In addition, occipital cortex GABA concentrations are increased after selective serotonin reuptake inhibitor administration (Sanacora et al., 2002). Furthermore, postmortem studies suggest that mood disorders are associated with a reduced number of GABAergic neurons in the frontal cortex (Heinz et al., 2001). The GABA(A) receptor is the site of action for certain anxiolytic drugs, including benzodiazepines and barbiturates, providing additional evidence for a role for the receptor in neuroticism. The Pro385Ser polymorphism is a Pro to Ser variant located in the a6 subunit of the GABA(A) receptor. Iwata et al., (1999) reported an association between this variant and benzodiazepine sensitivity. In addition, Schuckit et al., (1999) reported an association between Pro385Ser and low response to alcohol. Pro385Ser however, has yet to be investigated in personality traits, depression, or anxiety. In this study, we investigated the role of this polymorphism in Neuroticism, Extraversion, and Openness Personality Inventory (NEO-PI) personality traits, including neuroticism. To explore possible nonadditive genetic effects, we tested for potential statistical interactions between 
all variants that individually associate with neuroticism. In a previous report, a coding brain-derived neurotrophic factor (BDNF) variant was found to be associated with neuroticism in this sample (Sen et al., 2003). Thus, we explore interactions between variants that associate with neuroticism in this study, as well as potential interactions between each associated variant and BDNF with respect to neuroticism.

\section{Materials and Methods}

\section{Subjects}

The 242 female and 177 male subjects are from 257 families participating in the Family Blood Pressure Program at the Tecumseh, Michigan Site (Thiel et al., 2003). Subjects gave informed consent, and the study has been approved by the University of Michigan Medical School institutional review board. Ninety-nine percent of the subjects are non-Hispanic Caucasians. Family eligibility in this study required an available proband between 25 and 40 years of age with a systolic blood pressure in the upper $15 \%$ of the blood pressure distribution and a sibling willing to participate. Parents and additional siblings of probands were required when available.

\section{5-HTTLPR genotyping}

Primers SERT1 (5'-ATGCCAGCACCTAACCCCTAATGT$\left.3^{\prime}\right)$ and SERT2 (5'-GGACCGCAAGGTGGGCGGGA-3') were used to amplify a product that was 375 base pair (bp) product for the 14-repeat (s) allele and a $419 \mathrm{bp}$ product for the 16repeat (1) allele (Gelernter et al., 1998). A PTC 100 thermal cycler (MJ Research, Watertown, Massachusetts) was used for deoxyribonucleic acid (DNA) amplification. Amplification reactions were performed in a total volume of $20 \mu \mathrm{L}$, containing approximately $50 \mathrm{ng}$ of genomic template, $1 \mu \mathrm{mol} / \mathrm{L}$ of each primer, $200 \mu \mathrm{mol} / \mathrm{L}$ deoxynucleoside triphosphate (dNTP), 2 $\mu \mathrm{L}$ 10× Opti-Prime Buffer \#6 (Stratagene, La Jolla, California) and 1 unit of Taq polymerase. The polymerase chain reaction (PCR) cycling conditions consisted of an initial denaturation for $2 \mathrm{~min}$ at $94^{\circ} \mathrm{C}$, followed by 35 cycles of $94^{\circ} \mathrm{C}$ for $1 \mathrm{~min}, 60^{\circ} \mathrm{C}$ for $2 \mathrm{~min}$, and $72^{\circ} \mathrm{C}$ for $2 \mathrm{~min}$, and a final extension at $72^{\circ} \mathrm{C}$ for $4 \mathrm{~min}$. Polymerase chain reaction products were electrophoresed on a $2 \%$ agarose gel and visualized under ultraviolet light with the Gel-Star nucleic acid gel stain (BioWhittaker Molecular Applications, Rockland, Maine).

Originally, an alternate set of primers, STPR3 (5'-GGCGTTGCCGCTCTGAATTGC-3') and STPR5 (5'-GAGGGACTGAGCTGGACAACCCAC-3'), was used for amplification (Greenberg et al., 2000). These primers only amplified from the DNA of approximately $70 \%$ of subjects. In addition, in eight cases the genotypes produced were inconsistent with simple Mendelian inheritance, and the frequency of the genotypes produced with these primers did not fit into Hardy-Weinberg equilibrium. Upon reanalysis with the SERT1 and SERT2 primers, $91 \%$ of samples were successfully genotyped, and there were no Mendelian errors or deviation from Hardy Weinberg equilibrium. Comparison of the two methods showed that amplification with the STPR3 and STPR5 primers erroneously classified a subset of heterozygous $1 / \mathrm{s}$ subjects as s/s.

\section{Pro385Ser genotyping}

Two primers within the GABA A receptor a 6 (GABRA6) subunit gene, GABRA61, 5'-AGGCCAATAAAGTGCTCACG-3', and GABRA62, 5'-TTTACTGGTGCCTCCAAAGG-3', were used to amplify a $92 \mathrm{bp}$ PCR product from genomic DNA flanking the single nucleotide polymor- phism 1236C $>\mathrm{T}$ (Pro385-Ser). For genotyping, two molecular beacons were designed, one specific for the " $\mathrm{C}^{\text {" allele }}$ and $5^{\prime}$ labeled with a green fluorophore (FAM) (5'-/56-FAM/ AGCAGCAACCTGTCACACCCCCACCACTGCTGCT/ $\left.3 \mathrm{Dab} /-3^{\prime}\right)$ and the other specific for the " $\mathrm{T}$ " allele and 5 ' labeled with a red fluorophore (Texas red) (5'-/5TexRd-XN/ CTCCGGTCACCTGTCACATCCCCACCACACCGGAG/ $\left.3 \mathrm{Dab} /-3^{\prime}\right)$. Both oligonucleotides were 3' labeled with the quencher DABCYL. Molecular beacons were purchased from Integrated DNA Technologies, Coralville, Iowa. Genotyping was performed during real-time PCR in an iCycler Thermal Cycler (BioRad, Hercules, California). Amplification reactions were performed in a total volume of $25 \mu \mathrm{L}$ containing $10 \mathrm{pmol}$ each of the sense and antisense primers, $250 \mu \mathrm{mol} / \mathrm{L} \mathrm{dNTP,}$ $.34 \mathrm{~mol} / \mathrm{L}$ of each of the molecular beacons probes, 1 unit of Taq polymerase, and approximately $20 \mathrm{ng}$ of genomic DNA. The PCR conditions were as follows: initial denaturation for 3 min at $94^{\circ} \mathrm{C}$, followed by 35 cycles of $94^{\circ} \mathrm{C}$ for $30 \mathrm{sec}, 56^{\circ} \mathrm{C}$ for $1 \mathrm{~min}$, and $72^{\circ} \mathrm{C}$ for $30 \mathrm{sec}$. Fluorescence was measured during annealing temperature $\left(56^{\circ} \mathrm{C}\right)$. To verify the specificity of the molecular beacons as a single nucleotide polymorphism genotyping method, we analyzed a different subset of 56 samples through both molecular beacons and Fok I digestion. No discrepancies were found between the two methods.

\section{Questionnaire}

Personality traits were assessed with the NEO-PI. This inventory, consisting of 181 questions, assesses subjects on five global personality domains and breaks down three of these domains (neuroticism, extraversion, and agreeableness) into six facets each. The NEO-PI is a well-established inventory constructed through factor analytic strategies. This inventory also provides high test-retest reliability and longitudinal stability (Costa and McCrae, 1997).

Data to make diagnoses of alcohol abuse and dependence were not collected in this sample; however, subjects were asked questions regarding alcohol use. Specifically, subjects were asked to answer yes or no to the question: "Have you ever regularly consumed alcohol in your life?" $62.7 \%$ of subjects answered yes to this question. The presence of associations between genetic markers and subjects' response to this question were also explored.

\section{Statistical analysis}

The presence of association was determined with the QTDT Program version 2.1 (available at http://www.sph.umich. edu/statgen/abecasis/QTDT/). This program was not used to test for association through a transmission disequilibrium test. Instead, QTDT was used to test for association while accounting for familial resemblance due to kinship and linkage (Abecasis et al., 2000a, 2000b). The presence of a statistical interaction between the effect of two variants on neuroticism were assessed through linear regression in SPSS 10.0 (SPSS, Chicago, Illinois). The regression was performed on neuroticism with three independent factors: locus 1 genotype, locus 2 genotype, and the product of the two genotypes as an interaction term. Familial resemblance was not considered in testing for interactions.

\section{Results}

We determined genotypes for 419 subjects at the 5-HTTLPR polymorphic site. Allele frequencies in this sample (adjusted for familial correlations) were $\mathrm{S}=.42$ and $\mathrm{L}=.58$. 
Table I. NEO-PI Domain Scores as a Function of 5-HTTLPR and Pro385Ser Genotypes

\begin{tabular}{|c|c|c|c|c|c|c|c|c|}
\hline & Genotype & $n$ & Mean & SEM & $\begin{array}{c}\text { Regression } p \text { value } \\
\text { (2-tailed) }\end{array}$ & $\begin{array}{c}t \text { test } \\
\mathrm{s} \text { dominant }\end{array}$ & $\begin{array}{l}t \text { test } \\
\text { I dominant }\end{array}$ & $\begin{array}{r}\text { Regression } \\
\text { F statistic }\end{array}$ \\
\hline \multicolumn{9}{|l|}{ 5-HTTLPR } \\
\hline \multirow[t]{2}{*}{ Neuroticism } & $s / s$ & 83 & 87.8 & 2.07 & .008 & .006 & .024 & 9.00 \\
\hline & $1 / 1$ & 149 & 79.9 & 1.59 & & & & \\
\hline \multirow[t]{2}{*}{ Extraversion } & $s / s$ & 82 & 105.3 & 1.94 & ns & ns & ns & 1.45 \\
\hline & $s / l$ & 183 & 109.2 & 1.28 & & & & \\
\hline \multirow{2}{*}{ Openness } & $s / l$ & 183 & 104.4 & 1.26 & & & & \\
\hline & $\mathrm{I} / \mathrm{I}$ & 149 & 104.1 & 1.38 & & & & \\
\hline \multirow{3}{*}{ Conscientiousness } & $s / s$ & 83 & 48.0 & .88 & ns & ns & ns & .00 \\
\hline & $s / l$ & 184 & 48.6 & .62 & & & & \\
\hline & 1/I & 151 & 48.3 & .67 & & & & \\
\hline Agreeableness & $s / s$ & 83 & 45.4 & .72 & .049 & ns & .007 & 4.88 \\
\hline \multicolumn{9}{|l|}{ Pro385Ser } \\
\hline \multirow[t]{2}{*}{ Neuroticism } & Pro/Pro & 340 & 85.15 & 1.11 & .003 & & & 10.04 \\
\hline & Pro/Ser & 51 & 75.67 & 2.27 & & & & \\
\hline \multirow[t]{2}{*}{ Extraversion } & Pro/Pro & 340 & 108.07 & .95 & ns & & & 1.97 \\
\hline & Pro/Ser & 51 & 111.73 & 2.28 & & & & \\
\hline \multirow[t]{2}{*}{ Openness } & Pro/Pro & 339 & 103.63 & .93 & ns & & & 2.12 \\
\hline & Pro/Ser & 51 & 107.39 & 2.10 & & & & \\
\hline \multirow[t]{2}{*}{ Conscientiousness } & Pro/Pro & 343 & 48.25 & .45 & ns & & & .31 \\
\hline & Pro/Ser & 52 & 48.19 & 1.08 & & & & \\
\hline \multirow[t]{2}{*}{ Agreeableness } & Pro/Pro & 342 & 47.16 & .35 & ns & & & 1.13 \\
\hline & Pro/Ser & 52 & 48.18 & .93 & & & & \\
\hline \multirow[t]{2}{*}{ Alcohol Use } & Pro/Pro & 236 & .31 & .03 & ns & & & 1.27 \\
\hline & Pro/Ser & 35 & .40 & .08 & & & & \\
\hline
\end{tabular}

NEO-PI, Neuroticism, Extraversion, and Openness Personality Inventory; 5-HTTLPR, serotonin transporter promoter polymorphism.

These frequencies are consistent with reported values for samples with similar ethnic compositions: $\mathrm{S}=.45 ; \mathrm{L}=.55$ (Lesch et al., 1996).

In this sample, presence of the short allele is associated with a significantly higher mean neuroticism score $(p=.004)$. In addition, the presence of the 1 allele was associated with lower mean agreeableness $(p=.007)$. 5-HTTLPR alleles were not associated with the other three personality domains (Table 1). Of the six facets of neuroticism, 5-HTTLPR was associated with anxiety (N1), hostility (N2), depression (N3), and self consciousness (N4), but not with impulsiveness (N5) or vulnerability (N6) (Table 2). 5-HTTLPR was not associated with any of the 12 facets that compose the domains extraversion and openness. In the version of the NEO-PI used in this study, no facet scores for the agreeableness and conscientiousness domains were available. We also found an association between the 5-HTTLPR 1 allele and an increase in the presence of regular alcohol use $(p=.006)$ (Table 1$)$. A linear regression analysis showed no correlation between regular alcohol use and neuroticism in this sample $(p=.556)$.

Because previous studies are inconsistent concerning the dominance pattern at this locus (Greenberg et al., 2000; Melke et al., 2001), we did not assume any specific dominance pattern when testing association. For neuroticism, the s/s and 1/ s genotype groups did not differ significantly from each other, whereas both differed significantly from the 1/1 genotype group. For agreeableness, the $1 / 1$ and $s / 1$ groups did not dif- fer significantly from each other, whereas both groups differed significantly from the s/s group. For alcohol use, the s/s and 1/1 genotype groups differed significantly from each other, but neither homozygote group differed significantly from the s/ 1 group.

A total of 391 subjects were genotyped at the Pro385Ser polymorphism of GABRA6. Allele frequencies at this site (adjusted for familial correlations) were Pro $=.94$ and Ser $=.06$. This is consistent with reported allele frequencies (Pro $=.96$, Ser $=.04$; (Iwata et al., 1999). In our sample, there were no Ser/ Ser subjects. The Pro/Ser genotype was significantly associated with lower mean neuroticism but not with the other four personality domains (Table 1). Of the six facets of neuroticism, Pro385Ser was associated with anxiety (N1), hostility (N2), depression (N3), self consciousness (N4), and vulnerability (N6) (Table 2). In addition, Pro385Ser was associated with the extraversion facet called positive emotions (E6) and the openness facet called ideas (O5). Pro385Ser showed no association with the presence of regular alcohol use.

A total of 366 subjects were successfully genotyped at both the 5-HTTLPR and Pro385Ser sites. In the presence of the Pro/ Pro genotype, 5-HTTLPR was strongly associated with neuroticism (genotype: mean \pm SEM) (s/s: $90.98 \pm 2.61$; l/s: 86.75 \pm 1.79 ; $1 / 1$ : $80.07 \pm 1.69 ; p=.0004)$. In the presence of the alternate Pro/Ser genotype, 5-HTTLPR showed no association with neuroticism (s/s: $76.38 \pm 3.75 ; 1 /$ s: $75.43 \pm 3.70 ; 1 / 1: 75.27 \pm$ $4.28 ; p=.87)$. These results are illustrated in Figure 1 . In a lin- 


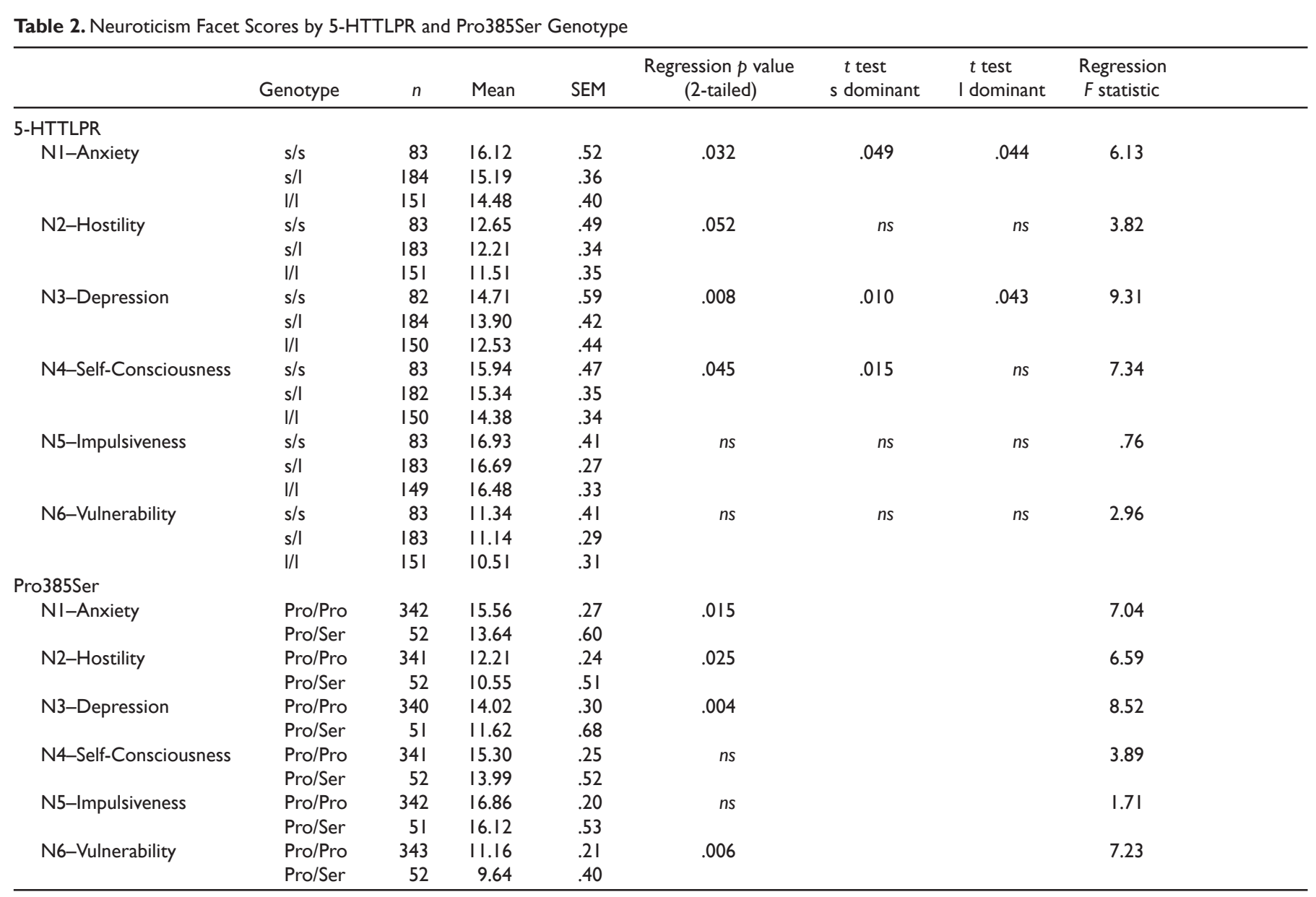

5-HTTLPR, serotonin transporter promoter polymorphism.

ear regression, the addition of the interaction term (5HTTLPR $\times$ Pro385Ser) was not significant $(p=.22)$.

In a previous report, we described an association between a BDNF polymorphism and neuroticism in this sample. There is no evidence of a statistical interaction between BDNF and either 5-HTTLPR $(p=.56)$ or GABRA6 $(p=.45)$ (graphs not shown but available upon request).

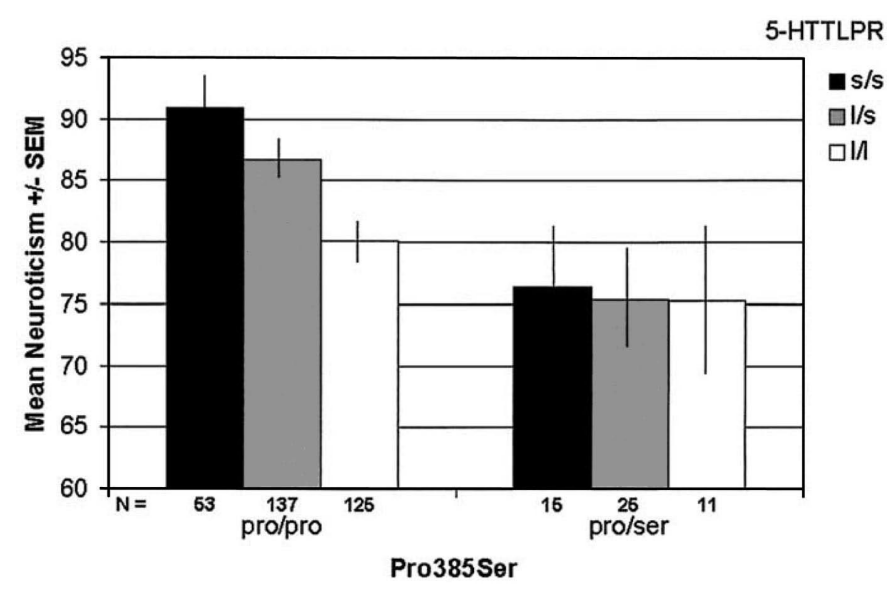

Figure I. Neuroticism scores by 5-HTTLPR and Pro385Ser genotypes in tandem. 5-HTTLPR, serotonin transporter promoter polymorphism.

\section{Discussion}

This study represents the first large-scale replication of the association between 5-HTTLPR and neuroticism described by a group other than Lesch et al. (1996). In our sample, 5-HTTLPR accounts for $2 \%-3 \%$ of the overall variance and $4 \%-5 \%$ of the genetic variance in neuroticism score. This is a smaller effect size than Lesch et al. (1996) found in the original study (overall variance: $3 \%-4 \%$; genetic variance: $7 \%-9 \%$ ), consistent with the finding that original reports of an association tend to overestimate the true effect size (Ioannidis et al., 2001).

There are several reasons why some previous studies might not have found an association between 5-HTTLPR and neuroticism. The most important is that the majority of studies investigating this association used samples that were too small to reliably detect this effect. Even with the effect size reported in the original study, power analysis shows that a sample of at least 350 subjects is necessary to obtain $80 \%$ statistical power at a .05 significance level (Flory et al., 1999). Of the 23 studies, 17 show associations in the predicted direction, indicating higher anxiety-related personality trait scores for subjects with the 5-HTTLPR s allele. In addition, whereas eight studies found significant evidence for an association between the 5-HTTLPR s allele and higher trait scores, not a single study found an association between the alternate 5-HTTLPR 1 allele and higher trait scores, arguing against a chance distribution. Of the studies in which a sample greater than 350 subjects was 
used, all three in which the NEO-PI was used found an association (our group used the NEO-PI; Lesch et al., [1996] and Greenberg et al., [2000] used the NEO-PI-R). In the three studies involving more than 350 subjects in which an association was not found, personality inventories other than the NEO-PI were used (Mazzanti et al., [1998] used the Tridimensional Personality Questionnaire; Hamer et al., [1999] used the Temperament and Character Inventory; Jorm et al., [1998] used the Eysenck Personality Questionnaire). Thus, 5-HTTLPR might be more closely associated to NEO-PI neuroticism than to other anxiety-related traits. In addition, our study, along with the two other large-scale positive association studies, used samples composed primarily of Caucasians not selected on the basis of psychopathology (Lesch et al., 1996; Greenberg et al., 2000); whether these findings are valid in other populations is still unknown. This report is also the first confirmation of an association between 5-HTTLPR and agreeableness by a group other than Lesch and colleagues. Our findings on agreeableness are in line with a recent study reporting significant covariance between agreeableness and neuroticism, $10 \%$ of which is attributable to the 5-HTTLPR locus (Jang et al., 2001).

The direction of association between 5-HTTLPR and neuroticism is the same in all studies finding significant association (s allele associates with higher mean neuroticism); however, which allele seems dominant differs among studies (Greenberg et al., 2000; Melke et al., 2001; this report). Similarly, the dominance pattern among expression studies is also inconsistent (Du et al., 1999; Hanna et al., 1998; Heils et al., 1995). In our study, apparent dominance of alleles at the 5-HTTLPR locus also shows different patterns for the different traits we analyzed in the same population sample. This heterogeneity in dominance patterns argues against complete dominance of either allele at this site, but further studies are warranted. Assuming the promoter variants affect expression, one would expect heterozygotes to show an intermediate phenotype, although not necessarily exactly at the midpoint. There are many reasons why not all studies show codominance. One possibility would be ceiling effects (heterozygotes and homozygotes showing close to maximal or close to the minimal scores) in a continuous scale such as the NEO-PI. Threshold effects on some but not all traits might also play a role.

In addition, we also report an association between the 5HTTLPR 1 allele and an increased presence of regular alcohol use. Schuckit et al. (1999) reported an association between the same allele and increased alcohol dependence. Regular alcohol use and alcohol dependence are distinct traits, so this study is not a strict replication of the findings presented by Schuckit et al; however, these two sets of results are likely related and should be explored further. Given that there is no association between neuroticism and regular alcohol use in this sample, the associations between 5-HTTLPR and these two traits are likely independent.

We also report an association between the Pro385Ser Pro/ Ser genotype and decreased neuroticism in this sample. Even though the two genotype groups differ markedly in mean neuroticism score, Pro385Ser accounts for only approximately $2 \%$ of the overall neuroticism variation and $4 \%$ of the genetic neuroticism variation in this sample, owing to the relatively low frequency of the Ser allele. This represents the first report of an association between Pro385Ser and a depression related trait. Schuckit et al. (1999) found that the Pro385Ser Pro/Ser genotype and the 5-HTTLPR 1/1 genotype associated with low response to alcohol and increased alcohol dependence. We find that the same two alleles at these loci are associated with lower neuroticism. Further studies are indicated to confirm the role of Pro385Ser in depression related traits, both individually and together with 5-HTTLPR.

The association between 5-HTTLPR and neuroticism was not corrected for multiple testing because it was designed as a replication of previously reported results. When a Bonferonni correction for the five NEO-PI domains is performed for Pro385Ser, the association with neuroticism remains significant. Because the neuroticism facets are strongly associated with each other, it is difficult to assess how to correct for further multiple testing.

Population stratification in studies such as ours can lead to false-positive associations (Lander and Schork, 1994). To assess this possibility, we used the program STRUCTURE, designed to infer population structure using genotypes from numerous unlinked markers as genomic controls (Pritchard and Rosenberg, 1999). Weak evidence for clustering into two groups was found ( $p=.024$; Theil and Schork, personal communication, February 15, 2002; data available upon request). For population structure to affect the results of an association test, the allele frequencies at the relevant loci and the trait score must differ between the two clusters. In our sample, there were no significant differences between the clusters in either 5-HTTLPR allele frequency $(p=.763)$, Pro385Ser allele frequency $(p=1.00)$, or neuroticism $(p$ $=.440$ ). Thus, these reported results are unlikely to be the product of population stratification. An additional concern for this study is that the sample was drawn from families with a moderately hypertensive proband. There is no association in this sample between blood pressure and either 5-HTTLPR or Pro385Ser and only a weak association with neuroticism $(p=.049)$. Nonetheless, the hypertensive status of the sample should be noted.

In addition to the individual associations between 5- HTTLPR and Pro385Ser with neuroticism, we also find suggestive evidence for an interaction between the two loci with reference to neuroticism. Specifically, the effect of 5-HTTLPR on neuroticism seems to be restricted to Pro385Ser Pro/Pro subjects. The interaction term did not achieve statistical significance in a linear regression analysis. This might be due to a lack of statistical power, in part because the Pro385Ser Pro/Ser group is relatively small $(n=51)$. The sample size needed to detect a significant statistical interaction is substantially larger than the sample needed to detect a simple association (Wahlsten, 1990). Although chance can of course not be excluded, it should be noted that this suggestive interaction is similar to one found by Schuckit et al. (1999). These authors found that all four subjects in their sample with both the 5-HTTLPR 1/ 1 genotype and the Pro/Ser genotype developed alcohol dependence and demonstrated a low level of response to alcohol. Given that Schuckit et al found a similar nonsignificant interaction in the same direction, the nature of this interaction with respect to neuroticism and alcoholism warrants further study in samples with large statistical power.

Little biological evidence directly connects the GABA A receptor a6 subunit and the serotonin transporter. There is evidence however, of interplay between the GABA and serotonin systems that might shed light on this statistical interaction. The majority of serotonergic axons in the brain synapse on cortical GABAergic interneurons (Smiley and Goldman-Rakic, 1996). In addition, recent evidence suggests that the convergence of serotonergic and dopaminergic neurons on GABAergic neurons might be important in the pathology of mood disorders (Benes and Berretta, 2001). The findings of Sanacora et al. (2002), that inhibitors of the serotonin transporter affect cortical GABA con- 
centrations, provides further evidence of a strong connection between the two neurotransmitter systems. The interplay between the two systems should be explored further to determine whether the suggestive interaction between 5-HTTLPR and Pro385Ser has a meaningful biological basis.

Neuroticism is a strong marker for vulnerability to depression (Duggan et al., 1995; Kendler et al., 1993). Characteristics of neuroticism make it particularly useful for genetic studies of depression. The reported heritability of neuroticism is 40\%-50\% (Jang et al., 1996; Lake et al., 2000), equal to or greater than heritibility estimates for depression (36\%) (Kendler and Prescott, 1999). Furthermore, approximately $70 \%$ of the correlation between neuroticism and depression risk is due to shared genetic risk factors (Kendler et al., 1993). Neuroticism is also a quantitative trait and stable through adulthood (Costa and McCrae, 1988). Our finding of associations between two polymorphisms and neuroticism suggest that these loci might contribute to the risk for depression.

\section{Acknowledgments}

This work was supported by the Nancy Pritzker Depression Research Network (RN, MB), the Michigan Society of Fellows (SS), the University of Michigan Medical Scientist Training Program Training Grant GM007863 (SS). Recruitment and DNA acquisition for the Tecumseh study was funded by National Heart Lung and Blood Institute Grant HL054512 (LG, AW).

We thank all the families who participated in the studies; Li Sheng, Goncalo Abecasis, Bonnie Theil, Nicholas Schork, and Maria Carolina Delgado for helpful discussions; and Aravinda Chakravarti for the DNA samples.

\section{References}

Abecasis et al., 2000a G. R. Abecasis, L. R. Cardon and W. O. Cookson, A general test of association for quantitative traits in nuclear families. Am J Hum Genet $\mathbf{6 6}$ (2000), pp. 279-292.

Abecasis et al., 2000b G. R. Abecasis, W. O. Cookson and L. R. Cardon, Pedigree tests of transmission disequilibrium. Eur J Hum Genet 8 (2000), pp. 545-55I.

Benes and Berretta, 200I F. M. Benes and S. Berretta, GABAergic interneurons: Implications for understanding schizophrenia and bipolar disorder. Neuropsychopharmacology 25 (200I), Pp. I-27.

Costa and McCrae, $1988<$ P.T. Costa, Jr. and R. R. McCrae, Personality in adulthood: A six-year longitudinal study of self-reports and spouse ratings on the NEO Personality Inventory.J Pers Soc Psychol 54 (1988), pp. 853-863.

Costa and McCrae, 1997 P. T. Costa, Jr. and R. R. McCrae, Stability and change in personality assessment: The revised NEO Personality Inventory in the year 2000.J Pers Assess 68 (1997), pp. 86-94.

Du et al., I999 L. Du, G. Faludi, M. Palkovits, E. Demeter, D. Bakish, Y. D. Lapierre et al., Frequency of long allele in serotonin transporter gene is increased in depressed suicide victims. Biol Psychiatry 46 (1999), pp. 196-201.

Duggan et al., $1995 \varangle$ C. Duggan, P. Sham, A. Lee, C. Minne and R. Murray, Neuroticism: A vulnerability marker for depression evidence from a family study.J Affect Disord 35 (1995), pp. I39-143.

Flory et al., 1999 J. D. Flory, S. B. Manuck, R. E. Ferrell, K. M. Dent, D. G. Peters and M. F. Muldoon, Neuroticism is not associated with the serotonin transporter (5HTTLPR) polymorphism. Mol Psychiatry 4 (1999), pp. 93-96.

Gelernter et al., 1998 J. Gelernter, H. Kranzler, E. F. Coccaro, L. J. Siever and A. S. New, Serotonin transporter protein gene polymorphism and personality measures in African American and European American subjects. Am J Psychiatry 155 (1998), Pp. 1332-1338.

Greenberg et al., 2000 B. D. Greenberg, Q. Li, F. R. Lucas, S. Hu, L.A. Sirota, J. Benjamin et al., Association between the serotonin transporter promoter polymorphism and personality traits in a primarily female population sample. Am J Med Genet 96 (2000), pp. 202-216.

Hamer et al., 1999 D. H. Hamer, B. D. Greenberg, S. Z. Sabol and D. L. Murphy, Role of the serotonin transporter gene in temperament and character. J Personal Disord 13 (1999), pp. 312-327.

Hanna et al., 1998 G. L. Hanna, J. A. Himle, G. C. Curtis, D. Q. Koram, J. VeenstraVanderWeele, B. L. Leventhal and E. H. Cook, Jr., Serotonin transporter and seasonal variation in blood serotonin in families with obsessive-compulsive disorder. Neuropsychopharmacology 18 (1998), pp. 102-1II (see comments).
Heils et al., 1995 A. Heils, A. Teufel, S. Petri, M. Seemann, D. Bengel, U. Balling et al., Functional promoter and polyadenylation site mapping of the human serotonin (5HT) transporter gene. J Neural Transm Gen Sect 102 ( I 995), pp. 247-254.

Heinz et al., 200I A. Heinz, K. Mann, D. R. Weinberger and D. Goldman, Serotonergic dysfunction, negative mood states, and response to alcohol. Alcohol Clin Exp Res 25 (200I), pp. 487-495.

loannidis et al., $200 \mathrm{I}$ J. P. loannidis, E. E. Ntzani, T. A. Trikalinos and D. G. Contopoulos-loannidis, Replication validity of genetic association studies. Nat Genet 29 (200I), pp. 306-309.

Iwata et al., I $999 \varangle$ N. Iwata, D. S. Cowley, M. Radel, P. P. Roy-Byrne and D. Goldman, Relationship between a GABAA alpha 6 Pro385Ser substitution and benzodiazepine sensitivity. Am J Psychiatry 156 (1999), pp. I447-I449.

Jang et al., 200 I K. L. Jang, S. Hu,W. J. Livesley,A.Angleitner, R. Riemann, J.Ando et al., Covariance structure of neuroticism and agreeableness: A twin and molecular genetic analysis of the role of the serotonin transporter gene. J Pers Soc Psychol 8 I (200I), pp. 295-304.

Jang et al., $1996<$ K. L. Jang,W. J. Livesley and P.A. Vernon, Heritability of the big five personality dimensions and their facets: A twin study.J Pers 64 (1996), pp. 577-591.

Jorm et al., $1998 \varangle$ A. F. Jorm, A. S. Henderson, P. A. Jacomb, H. Christensen, A. E. Korten, B. Rodgers et al., An association study of a functional polymorphism of the serotonin transporter gene with personality and psychiatric symptoms. Mol Psychiatry 3 (1998), pp. 449-45।.

Kendler et al., $1993 \varangle$ K. S. Kendler, M. C. Neale, R. C. Kessler, A. C. Heath and L. J. Eaves, A longitudinal twin study of personality and major depression in women. Arch Gen Psychiatry 50 (1993), pp. 853-862.

Kendler and Prescott, $1999<$ K. S. Kendler and C. A. Prescott, A population-based twin study of lifetime major depression in men and women. Arch Gen Psychiatry 56 (1999), pp. 39-44.

Lake et al., $2000 \varangle$ R. I. Lake, L. J. Eaves, H. H. Maes, A. C. Heath and N. G. Martin, Further evidence against the environmental transmission of individual differences in neuroticism from a collaborative study of 45,850 twins and relatives on two continents. Behav Genet 30 (2000), pp. 223-233.

Lander and Schork, $1994 \varangle$ E. S. Lander and N. J. Schork, Genetic dissection of complex traits. Science 265 (1994), pp. 2037-2048.

Lesch et al., 1996 K. P. Lesch, D. Bengel, A. Heils, S. Z. Sabol, B. D. Greenberg, S. Petri et al., Association of anxiety-related traits with a polymorphism in the serotonin transporter gene regulatory region. Science 274 (1996), pp. |527-I53|.

Little et al., 1998 K.Y. Little, D. P. McLaughlin, L. Zhang, C. S. Livermore, G.W. Dalack, P. R. McFinton et al., Cocaine, ethanol, and genotype effects on human midbrain serotonin transporter binding sites and mRNA levels. Am J Psychiatry I55 (1998), pp. 207-213.

Mazzanti et al., 1998 C. M. Mazzanti, J. Lappalainen, J. C. Long, D. Bengel, H. Naukkarinen, M. Eggert et al., Role of the serotonin transporter promoter polymorphism in anxiety-related traits. Arch Gen Psychiatry 55 (1998), pp. 936-940.

Melke et al., 200I J. Melke, M. Landen, F. Baghei, R. Rosmond, G. Holm, P. Bjorntorp et al., Serotonin transporter gene polymorphisms are associated with anxiety-related personality traits in women. Am J Med Genet 105 (200I), pp. 458-463.

Petty, $1995 \varangle$ F. Petty, GABA and mood disorders: A brief review and hypothesis. J Affect Disord 34 (1995), pp. 275-28I.

Pritchard and Rosenberg, $1999<\mathrm{J}$. K. Pritchard and N. A. Rosenberg, Use of unlinked genetic markers to detect population stratification in association studies. Am J Hum Genet 65 (1999), pp. 220-228.

Sanacora et al., $2000 \varangle$ G. Sanacora, G. F. Mason and J. H. Krystal, Impairment of GABAergic transmission in depression: New insights from neuroimaging studies. Crit Rev Neurobiol 14 (2000), pp. 23-45.

Sanacora et al., 1999 G. Sanacora, G. F. Mason, D. L. Rothman, K. L. Behar, F. Hyder, O. A. Petroff et al., Reduced cortical gamma-aminobutyric acid levels in depressed patients determined by proton magnetic resonance spectroscopy. Arch Gen Psychiatry 56 (1999), pp. 1043-1047.

Sanacora et al., $2002 \varangle$ G. Sanacora, G. F. Mason, D. L. Rothman and J. H. Krystal, Increased occipital cortex GABA concentrations in depressed patients after therapy with selective serotonin reuptake inhibitors. Am J Psychiatry 159 (2002), PP. 663-665.

Schuckit et al., $1999 \varangle$ M. A. Schuckit, C. Mazzanti, T. L. Smith, U. Ahmed, M. Radel, N. Iwata and D. Goldman, Selective genotyping for the role of 5-HT2A, 5-HT2C, and GABA alpha 6 receptors and the serotonin transporter in the level of response to alcohol:A pilot study. Biol Psychiatry 45 (1999), pp. 647-65I.

Sen et al., $2003 \varangle$ S. Sen, R. M. Nesse, S. F. Stoltenberg, S. Li, L. Gleiberman, A. Chakravarti et al., A BDNF coding variant is associated with the NEO personality inventory domain neuroticism, a risk factor for depression. Neuropsychopharmacology 28 (2003), pp. 397-401.

Smiley and Goldman-Rakic, $1996<$ J. F. Smiley and P. S. Goldman-Rakic, Serotonergic axons in monkey prefrontal cerebral cortex synapse predominantly on interneurons as demonstrated by serial section electron microscopy.J Comp Neurol 367 (1996), pp. 43I-443.

Thiel et al., 2003 B. A. Thiel, A. Chakravarti, R. S. Cooper, A. Luke, S. Lewis, A. Lynn et al., A genome-wide linkage analysis investigating the determinants of blood pressure in whites and African Americans. Am J Hypertens 16 (2003), pp. I5I-I53.

Wahlsten, $1990 \varangle \mathrm{D}$. Wahlsten, Insensitivity of the analysis of variance to heredity-environment interaction. Behav Brain Sci 13 (1990), pp. 109-16I. 